\title{
Flowing with the temporal renormalization group
}

\author{
Lukas Corell, ${ }^{1}$ Anton K. Cyrol $\odot,{ }^{1}$ Markus Heller $\odot,{ }^{1}$ and Jan M. Pawlowski ${ }^{1,2}$ \\ ${ }^{1}$ Institut für Theoretische Physik, Universität Heidelberg, \\ Philosophenweg 16, 69120 Heidelberg, Germany \\ ${ }^{2}$ ExtreMe Matter Institute EMMI, GSI, Planckstraße 1, 64291 Darmstadt, Germany
}

(Received 16 April 2020; accepted 8 June 2021; published 6 July 2021)

\begin{abstract}
We discuss the far-from-equilibrium evolution of $\phi^{3}$ theory in $1+1$ dimensions with the temporal functional renormalization group. In particular, we show that this manifestly causal approach leads to novel one-loop exact equations for fully dressed correlation functions. Within this setup, we numerically compute the dynamical propagator. Its behavior suggests self-similarity far from equilibrium in a restricted momentum regime. We discuss the scaling exponents for our solution, as well as the numerical satisfaction of energy and particle number conservation. We also derive a simple exact representation of the expectation value of the energy-momentum tensor solely in terms of the propagator.
\end{abstract}

DOI: $10.1103 /$ PhysRevD.104.025005

\section{INTRODUCTION}

The dynamics of quantum systems far from equilibrium has received much attention in the past decades. The theoretical resolution of these processes is crucial for the understanding of systems reaching from early universe cosmology and heavy ion collisions to the dynamics of ultracold atom clouds in table top experiments. This has triggered many theoretical developments ranging from lattice to diagrammatic approaches; for reviews, see, e.g., [1-3].

In the present work, we apply and develop further a functional renormalization group (fRG) approach [4-6] to nonequilibrium physics put forward in $[7,8]$ based on a temporal cutoff. For related developments in the context of cosmology, see also [9]; for nonequilibrium fRG applications with a standard momentum cutoff, see, e.g., [10-27].

The formulation of the cutoff in momentum space leads to a modification of local conservation laws reaching from conserved charges to gauge theories. While this modification is well captured within modified symmetry identities, the control of such modifications is even more important for the dynamics of a system. There, a violation of conservation laws can cause secularities and hence a breakdown of the approach at hand. This has triggered the development of a fRG approach for quantum dynamics based on a (temporal) cutoff of the Schwinger-Keldysh contour $[7,8]$. This cutoff simply suppresses the time evolution of a system beyond the cutoff time $\tau$. Trivially,

Published by the American Physical Society under the terms of the Creative Commons Attribution 4.0 International license. Further distribution of this work must maintain attribution to the author(s) and the published article's title, journal citation, and DOI. Funded by SCOAP ${ }^{3}$. the temporal flow of the system with this cutoff time is manifestly causal, and the flow equation captures the time evolution of the system at $t=\tau$. Apart from its manifest causality, its formulation in position space preserves all local conservation laws including gauge symmetries. In summary, it is the manifest preservation of causality and of local symmetries which is at the root of the temporal fRG (t-fRG) approach.

In the present work, we further develop the t-fRG approach as well as applying it to the dynamics of a $\phi^{3}$ theory in $1+1$ dimensions. Such a theory is an ideal test case for the present approach, but it is also of interest for extending the far-from-equilibrium universality known from $\phi^{4}$ interactions (relativistic and nonrelativistic, e.g., [28]) and gauge theories to the $\phi^{3}$ theory. Furthermore, it is interesting with regard to non-Abelian gauge theories that have both microscopic three-field and four-field vertices.

In Sec. II, we briefly review the temporal fRG and develop it further. This leads us to integrated flow equations, which are novel one-loop exact dynamical relations for the full unregularized correlation functions. In Sec. III, we apply the approach to the $\phi^{3}$ theory in $1+1$ dimensions and compute the dynamics of the propagator. We also derive simple relations for the expectation value of the energy-momentum tensor solely in terms of the propagator. We discuss the self-similar scaling behavior of the propagator as well as the numerical satisfaction of energy and particle number conservation. Our results are briefly summed up in Sec. IV.

\section{TEMPORAL FUNCTIONAL RENORMALIZATION GROUP}

In this section, we briefly review the t-fRG approach; for more details, see $[7,8]$. We discuss further formal 
developments that are also important for the present numerical application. In particular, we derive one-loop exact functional relations for the full (cutoff independent) correlation functions of a given quantum theory by simply integrating the temporal cutoff parameter.

\section{A. Closed time path}

In the context of nonequilibrium phenomena, it is instructive to employ a real-time formalism. We choose to work in the Schwinger-Keldysh formalism, which was developed in [29-34]. In this section, we introduce this approach and discuss its basic properties with regard to the time evolution of correlation functions.

At first, we consider the density matrix $\rho(t)$ which contains all information on the state of a quantum system at a time $t$. Usually, the density matrix is known at some initial time $t_{0}$ at which we prepare our system. It could very well describe a system in thermal equilibrium, where $\rho_{0}=\rho\left(t_{0}\right) \propto e^{-\beta H}$ with the inverse temperature $\beta$ and the Hamiltonian $H$. Generally, it describes any system and, in particular, interesting ones far-from-equilibrium. The time evolution of the density matrix is governed by the unitary time evolution operator $U$ and we write

$$
\rho(t)=U\left(t, t_{0}\right) \rho\left(t_{0}\right) U\left(t_{0}, t\right) .
$$

Typically, we are interested in the time evolution of an operator expectation value $\langle\mathcal{O}\rangle(t)$, e.g., a correlation function. For any operator, the expectation value at time $t$ is given as the trace over the density matrix and the operator

$$
\langle\mathcal{O}\rangle(t)=\operatorname{Tr}[\rho(t) \mathcal{O}]
$$

To work out a more illustrative form of this expectation value, we use (1) and exploit that the trace is invariant under cyclic permutations of the operators to find

$$
\langle\mathcal{O}\rangle(t)=\operatorname{Tr}\left[U\left(t_{0}, t\right) \mathcal{O} U\left(t, t_{0}\right) \rho\left(t_{0}\right)\right] .
$$

Reading the argument of the trace from right to left, the initial density matrix is evolved in time from $t_{0}$ to $t$ where the operator $\mathcal{O}$ is inserted. Subsequently, there is a time evolution back to the initial time. This operator ordering, which starts and ends at the same time, directly suggests the term closed time path (CTP) which is used in the Schwinger-Keldysh formalism. A representation of the closed time path for the expectation value $\mathcal{O}(t)$ is shown in Fig. 1.

It is now possible to extend the closed time path to infinity by using the unitarity of the time evolution operator. This is accomplished by inserting $U(t, \infty) U(\infty, t)=\mathbb{1}$ either to the left or the right of the operator in (3). We denote this extended path with $\mathcal{C}$. Depending on which side we extend the time path, the operator is regarded as being placed on the

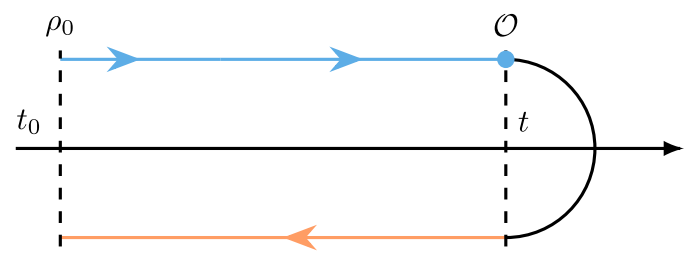

FIG. 1. Closed time path used to compute the time-dependent expectation value of the operator $\mathcal{O} . \rho_{0}$ is the density matrix at the initial time $t_{0}$. Note that the time path does not need to extend further than the latest operator insertion.

forward or backward branch of the CTP. Due to the construction, it is clear that there is no difference for a single operator.

As soon as we insert multiple operators, it is crucial where each one is placed. This is incorporated into the formalism by considering contour-time-ordered correlation functions. This time ordering on the closed time path is best understood as walking along the path in the direction of the arrow in Fig. 1. Thus, time ordering on the forward branch is the usual time ordering, while on the backward branch, it is antitime ordering. In particular, this means that every time on the backward part of the contour is considered later than any time on the forward part.

Now, we define the generating functional for nonequilibrium correlation function

$$
Z[J ; \rho]=\operatorname{Tr}\left[\rho\left(t_{0}\right) \mathcal{T}_{\mathcal{C}} \exp \left\{\mathrm{i} \int_{\mathcal{C}(x)} \varphi(x) J(x)\right\}\right]
$$

where we introduced the notation $\int_{\mathcal{C}(x)}=\int_{\mathcal{C}} \mathrm{d} x^{0} \int_{\mathbb{R}^{d}} \mathrm{~d}^{d} x$, the contour-time-ordering operator $\mathcal{T}_{\mathcal{C}}$, and the source $J$. Correlation functions are now obtained as functional derivatives with respect to the source

$$
\left\langle\varphi\left(x_{1}\right) \cdots \varphi\left(x_{n}\right)\right\rangle=\left.(-\mathrm{i})^{n} \frac{\delta^{n} Z[J ; \rho]}{\delta J\left(x_{1}\right) \cdots \delta J\left(x_{n}\right)}\right|_{J=0} .
$$

For more details, see $[7,8]$.

\section{B. Flow equation}

Consider now a CTP that only extends to some finite (cutoff) time $\tau$. The corresponding generating functional $Z_{\tau}[J ; \rho]$ only sums over fluctuations up to $\tau$. Therefore, it contains no sources for quantum fluctuations for times later than $\tau$. Causality entails that all $n$-point functions derived from $Z_{\tau}$ are the full correlation functions as derived from (4), if all time arguments $x_{i}^{0}$ with $i=1, \ldots, n$ obey $x_{i}^{0} \leq \tau$. See Fig. 2 for a graphical representation.

In turn, if at least one time argument of the $n$-point function is larger than $\tau$, the correlator vanishes identically. Figure 3 shows such a scenario.

We now turn to the effective action $\Gamma_{\tau}$, the generating functional of 1PI (one-particle-irreducible) correlation 


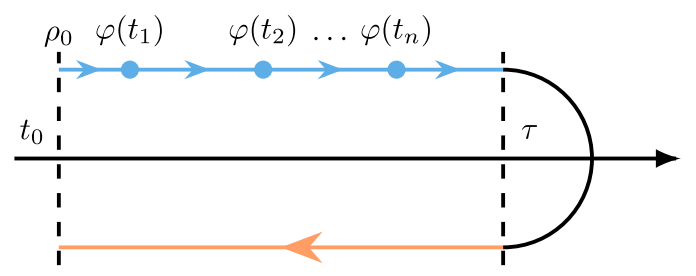

FIG. 2. n-point function on the closed time path with the latest time $t_{n}<\tau$. Hence, all fluctuations up to $t_{n}$ are included and we obtain the fully dressed correlation function.

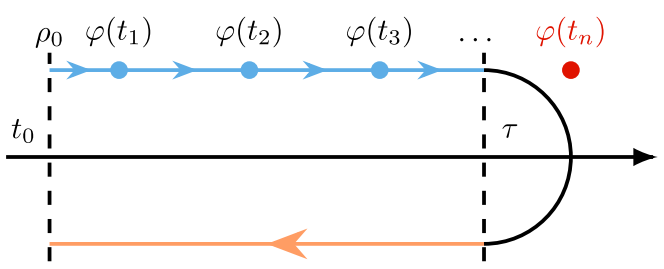

FIG. 3. n-point function on the closed time path with the latest time $t_{n}>\tau$. Such fluctuations are not included in the generating functional $Z_{\tau}$. Thus, this correlator vanishes identically.

functions with time arguments smaller than or equal to $\tau$. To that end, we consider the Schwinger functional

$$
W_{\tau}[J ; \rho]=-\mathrm{i} \ln Z_{\tau}[J ; \rho],
$$

which inherits the causal properties from $Z_{\tau}$. Finally, we are interested in moving the cutoff time $\tau$ to infinity. For deriving a flow equation for the generating functionals, it is more convenient to implement the finite cutoff time via regulator terms as also used for standard momentum cutoffs in the fRG. Hence, we add a cutoff term to the classical action

$$
\Delta S_{\tau}[\varphi]=\frac{1}{2} \int_{\mathcal{C}(x, y)} \varphi(x) R_{\tau}(x, y) \varphi(y)
$$

We demand that the cutoff term (7) vanishes for both times $x^{0}, y^{0} \leq \tau$ and suppresses all fluctuations for later times. The respective regulator $R_{\tau}$ is specified later in Sec. II C. Such a term implements the discussed causal properties. The effective action is then obtained as the Legendre transformation

$$
\begin{aligned}
\Gamma_{\tau}[\phi ; \rho]= & W_{\tau}[J ; \rho]-\int_{\mathcal{C}(x)} \phi(x) J(x) \\
& -\frac{1}{2} \int_{\mathcal{C}(x, y)} \phi(x) R_{\tau}(x, y) \phi(y),
\end{aligned}
$$

with the field expectation value $\phi(x)=\langle\varphi(x)\rangle$. Completely analogously to the equilibrium case, we can derive a flow equation for the effective action

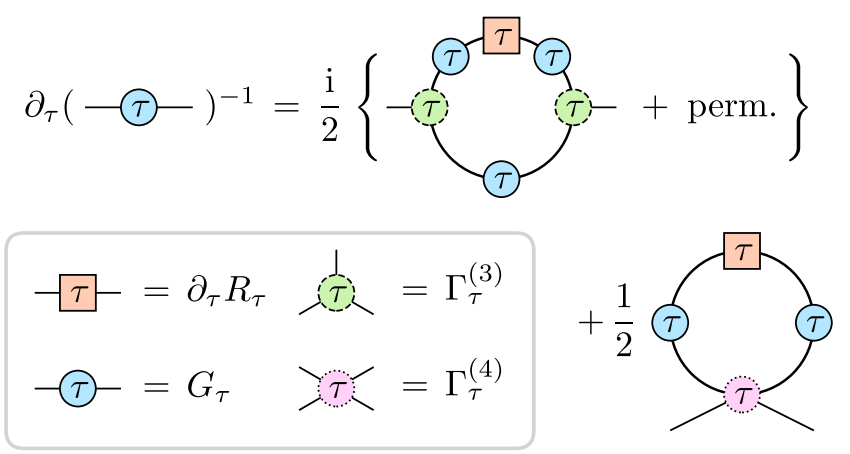

FIG. 4. Diagrammatic representation of the flow equation of the two-point function. The orange square denotes $\partial_{\tau} R_{\tau}(x, y)$. Lines with blue circles represent the fully dressed propagator $G_{\tau}(x, y)=\mathrm{i}\left[\Gamma_{\tau}^{(2)}+R_{\tau}\right]^{-1}(x, y)$. The green circle with three lines attached is $\Gamma_{\tau}^{(3)}$ and the pink circle with four legs is $\Gamma_{\tau}^{(4)}$, both also fully dressed. perm. stands for the permutation of the external legs.

$$
\partial_{\tau} \Gamma_{\tau}[\phi]=\frac{1}{2} \int_{\mathcal{C}(x, y)} G_{\tau}(x, y) \partial_{\tau} R_{\tau}(x, y) .
$$

A detailed derivation can be found in [8]. This flow equation successively integrates out all relevant fluctuations time slice by time slice, thus providing us with the time evolution of the considered system. The regularized propagator $G_{\tau}$ is given by

$$
G_{\tau}(x, y)=-\left.\mathrm{i} \frac{\delta^{2} W_{\tau}[J ; \rho]}{\delta J(x) \delta J(y)}\right|_{J=0}
$$

and is related to the 1PI two-point function by

$$
\mathrm{i} G_{\tau}^{-1}(x, y)=\left[\Gamma_{\tau}^{(2)}+R_{\tau}\right](x, y) .
$$

Flow equations for the 1PI correlation functions $\Gamma^{(n)}$ are obtained by taking functional derivatives with respect to the field expectation value

$$
\Gamma_{\tau}^{(n)}\left(x_{1}, \ldots, x_{n}\right)=\frac{\delta^{n} \Gamma_{\tau}}{\delta \phi\left(x_{1}\right) \cdots \delta \phi\left(x_{n}\right)}
$$

Analogously to the flow equation in momentum space, this yields an infinite hierarchy of coupled differential equations for the 1PI correlation functions. To solve it, this hierarchy has to be truncated.

For now, we focus on the flow equation of the two-point function, which is depicted in Fig. 4. The full, i.e., not truncated, flow reads 


$$
\begin{aligned}
\partial_{\tau} \Gamma_{\tau}^{(2)}(x, y) \\
=\frac{\mathrm{i}}{2} \int_{\mathcal{C}\left(z_{1}, z_{2}\right)} \Gamma_{\tau}^{(4)}\left(x, y, z_{1}, z_{2}\right)\left(G_{\tau} \partial_{\tau} R_{\tau} G_{\tau}\right)\left(z_{2}, z_{1}\right) \\
\quad-\frac{1}{2} \int_{\mathcal{C}\left(z_{1}, \ldots, z_{4}\right)}\left\{\Gamma_{\tau}^{(3)}\left(x, z_{1}, z_{2}\right) G_{\tau}\left(z_{2}, z_{4}\right) \Gamma_{\tau}^{(3)}\left(z_{3}, z_{4}, y\right)\right. \\
\left.\quad \times\left(G_{\tau} \partial_{\tau} R_{\tau} G_{\tau}\right)\left(z_{1}, z_{3}\right)+\text { perm. }\right\} .
\end{aligned}
$$

It depends not only on the propagator $G_{\tau}$, but also on $\Gamma_{\tau}^{(3)}$ and $\Gamma_{\tau}^{(4)}$. We discuss the truncation we used to solve it in Sec. III.

The fact that higher-order correlation functions enter in the flow of the lower-order ones is a general feature of fRG equations. Typically, the flow of $\Gamma_{\tau}^{(n)}$ contains contributions from $\Gamma_{\tau}^{(n+1)}$ and $\Gamma_{\tau}^{(n+2)}$. In particular, non-Gaussian initial conditions are implemented with nontrivial $\Gamma_{\tau=t_{0}}^{(n>2)}$ at the initial time $t_{0}$.

\section{Causality}

We implicitly defined the regulator by requiring that no information after the cutoff time $\tau$ can contribute to correlation functions with time arguments earlier than $\tau$. This does not determine the regulator uniquely but a simple and useful choice is given by the sharp regulator

$$
\mathrm{i} R_{\tau}(x, y)=\delta_{\mathcal{C}}(x-y)\left\{\begin{array}{ll}
\infty & \text { if } x_{0}=y_{0}>\tau \\
0 & \text { otherwise }
\end{array} .\right.
$$

The delta distribution $\delta_{\mathcal{C}}$ on the closed time path is defined, so that

$$
\int_{\mathcal{C}(y)} \delta_{\mathcal{C}}(x-y) f(y)=f(x)
$$

A particular important consequence of causality is that the regulated propagator $\langle\varphi(x) \varphi(y)\rangle_{1 \mathrm{PI}}$ is strictly zero if at least one time argument exceeds the cutoff time. In turn, for vanishing or constant backgrounds $\phi_{c}$, it is the fully interacting propagator $G \equiv G_{\tau=\infty}$ otherwise. We write the regularized propagator as

$$
G_{\tau}(x, y)=G(x, y) \theta\left(\tau-x^{0}\right) \theta\left(\tau-y^{0}\right) .
$$

Equation (15) entails the important property that the full propagator for vanishing or constant fields $\phi_{c}$ only depends on the full cutoff-independent two-point function $\Gamma^{(2)}\left[\phi_{c}\right]$ at $\tau=\infty$ with

$$
\Gamma^{(n)}[\phi]=\Gamma_{\tau=\infty}^{(n)}[\phi] .
$$

Note that for general space-time-dependent backgrounds, this cannot hold true as such backgrounds can have support for all times.
The surprising property (15) is deeply rooted in the locality and causality of the present cutoff procedure. Furthermore, it is linked to the functional optimization of the fRG [35]. There, it has been shown that optimized fRG flows have a related property: for optimal cutoffs, the regulator variation of the two-point function perpendicular to the direction of the optimized flow vanishes: $\delta_{\perp} \Gamma_{k}^{(2)}=0$. The local temporal regularization discussed in the present work shares this property.

A useful alternative representation of (15) is given by

$$
G_{\tau}(x, y)=\left[\frac{\mathrm{i}}{\Gamma^{(2)}+R_{\tau}}\right](x, y) \text {. }
$$

The causal structure extends to all correlation functions $\left\langle\varphi\left(x_{1}\right) \cdots \varphi\left(x_{n}\right)\right\rangle_{1 \mathrm{PI}}$ : they are fully dressed as long as all their time arguments are smaller or equal to $\tau$ and the initial ones otherwise. Hence, $\Gamma_{\tau}^{(n)}\left[\phi_{c}\right]$ for constant background $\phi_{c}$ with $n \geq 2$ can be written as

$$
\begin{aligned}
& \Gamma_{\tau}^{(n)}\left(x_{1}, \ldots, x_{n}\right) \\
& \quad=\Gamma_{t_{0}}^{(n)}\left(x_{1}, \ldots, x_{n}\right)+\Delta \Gamma^{(n)}\left(x_{1}, \ldots, x_{n}\right) \prod_{i=1}^{n} \theta\left(\tau-x_{i}^{0}\right),
\end{aligned}
$$

with

$$
\Delta \Gamma^{(n)}=\Gamma^{(n)}-\Gamma_{t_{0}}^{(n)} .
$$

For more details, see also Appendix B of [8]. These are the causal properties discussed in Sec. II A in terms of 1PI correlators. They are preserved by the flow equation and make this approach manifestly causal.

The causality constraints of the present temporal fRG also lead to another very important identity that is peculiar to our approach: for constant backgrounds, the line with the cutoff insertion $G_{\tau} \cdot \partial_{\tau} R_{\tau} \cdot G_{\tau}$ is simply given by the $\tau$ derivative of the propagator,

$\partial_{\tau} G_{\tau}(x, y)=\mathrm{i} \int_{\mathcal{C}\left(z_{1}, z_{2}\right)} G_{\tau}\left(x, z_{1}\right) \partial_{\tau} R_{\tau}\left(z_{1}, z_{2}\right) G_{\tau}\left(z_{2}, y\right)$.

Equation (20) follows readily from the $\tau$ derivative of (15) using the representation (17). In contrast to standard flows with momentum cutoffs, the term proportional to $\partial_{\tau} \Gamma_{\tau}^{(2)}$ is absent. Equation (20) has important implications on the general structure of the temporal flow equations and is crucial for the approach.

We emphasize that the renormalization of the theory is done for the initial state, or more precisely, for the correlation functions at the initial time. In contradistinction to time-dependent renormalization procedures, such a timeindependent one naturally incorporates the underlying RG invariance. More details will be presented in $[36,37]$. 


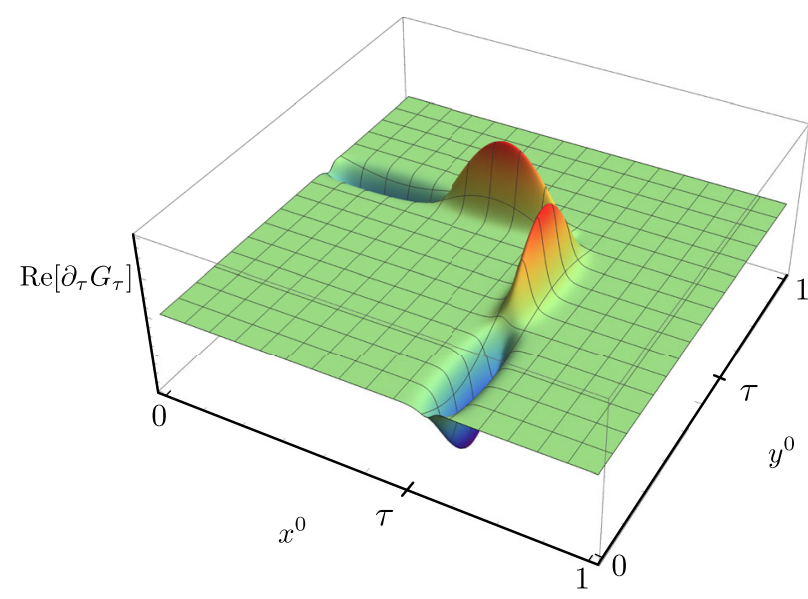

FIG. 5. Illustration of the causal structure of the propagator derivative $\partial_{\tau} G_{\tau}$ [cf. (21)]. For better visualization, we introduced a finite width to the contributing $\theta$ and $\delta$ functions. The oscillating structure is the real part of the free propagator (both field operators are inserted on the forward branch of the CTP but other insertions give a similar picture). The imaginary part basically only differs by a phase.

\section{Integrated flow}

The causality of the flow equation, in particular, the properties of the propagator (15), and the higher 1PI correlation functions (20), as well as the relation for the regulator derivative (20), have the remarkable consequence that the time flow can always be integrated analytically.

This integration is possible since all flow equations for the $\Gamma_{\tau}^{(n)}$ can only contain the regulator derivative in the form of (20) which can be replaced by the $\tau$ derivative of the propagator,

$$
\begin{aligned}
\partial_{\tau} G_{\tau}(x, y)= & G(x, y)\left[\delta\left(\tau-x^{0}\right) \theta\left(\tau-y^{0}\right)\right. \\
& \left.+\theta\left(\tau-x^{0}\right) \delta\left(\tau-y^{0}\right)\right] .
\end{aligned}
$$

A visualization of (21) is depicted in Fig. 5. For illustration purposes, we introduced a finite width for the $\theta$ and $\delta$ functions and plugged in the free propagator for $G$. In Fig. 5, we show the real part of the free propagator (both field operators are inserted on the forward branch of the CTP but other insertions give a similar picture). The imaginary part basically only differs by a phase.

Now, consider the first line of (21). At $x^{0}=\tau$, the propagator derivative in $y^{0}$ direction carries the oscillating shape of the real part of the free propagator. However, as soon as $y^{0}>\tau$, the propagator vanishes. The same holds if we swap $x^{0}$ and $y^{0}$.

Taking a $\tau$ derivative of (18), we obtain relations similar to (21) for the $\Gamma_{\tau}^{(n)}$. Therefore, the full $\tau$ dependence of the flow equations is captured by $\theta$ and $\delta$ distributions. This guarantees that the $\tau$ integration can always be performed analytically.
Strikingly, the resulting equations are one-loop equations for the fully dressed correlation functions. This is particularly useful when the theory has to be renormalized as the divergent contributions are readily identified. Further note that this is really due to causality. For example, a sharp cutoff regulator in momentum space does not lead to an integrated flow that is one loop.

We demonstrate the analytic integration for the case of the two-point function in Appendix A.

\section{DYNAMICS OF THE $\phi^{3}$ THEORY}

In this section, we implement the t-fRG approach for a scalar field with cubic interaction in $1+1$ dimensions with the classical action

$S[\varphi]=\int_{\mathcal{C}(x)}\left\{\frac{1}{2} \partial^{\mu} \varphi(x) \partial_{\mu} \varphi(x)-\frac{m^{2}}{2} \varphi(x)^{2}-\frac{\lambda}{3 !} \varphi(x)^{3}\right\}$.

As discussed in Sec. I, this theory is an ideal test case for the present approach. Moreover, microscopic cubic interactions are also present in non-Abelian gauge theories. Even though the latter are momentum dependent, the scalar field theory with (22) allows for the same scattering processes. Since those scattering processes are absent in the $\phi^{4}$ theory, the insights from cubic interactions are a necessity in regard to non-Abelian gauge theories.

\section{A. Truncated flow for the propagator}

In the present work, we discuss the dynamics of the propagator with classical three-point functions. In contradistinction to the $\phi^{4}$ theory, in the $\phi^{3}$ theory this already gives rise to a nontrivial dynamical evolution. The vertex reads

$$
\begin{aligned}
\Gamma_{\tau}^{(3)}\left(x, z_{1}, z_{2}\right) & =S^{(3)}\left(x, z_{1}, z_{2}\right) \\
& =-\lambda \delta_{\mathcal{C}}\left(x-z_{1}\right) \delta_{\mathcal{C}}\left(z_{1}-z_{2}\right) .
\end{aligned}
$$

Additionally, we set $\Gamma_{\tau}^{(n)}=0$ for all $n>3$. This leads us to the truncated flow equation for the two-point function shown in Fig. 6. In this approximation, we study the emergence of universal dynamics in the $\phi^{3}$ theory. This universal dynamics can be generated with Gaussian initial conditions (see previous 2PI studies of $\phi^{4}$ theory, e.g., $[38,39])$.

Let us emphasize again that including non-Gaussian initial conditions is straightforward in the $\mathrm{t}$-fRG framework in the form of $\Gamma_{\tau=t_{0}}^{(n>2)}$. The respective higher order correlations are, e.g., part of a thermal state given by a density matrix of the form $\rho \propto \exp ^{-\beta H}$ with the Hamiltonian $H$. Hence, the Gaussian initial state maybe understood as the result of some type of quench which drives the system out of equilibrium. 


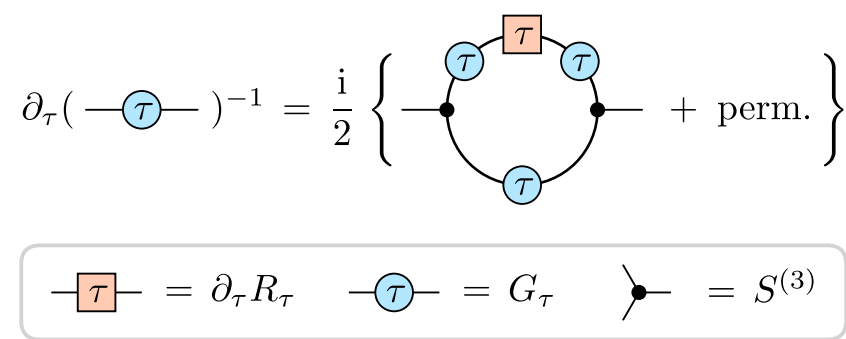

FIG. 6. Truncated flow equation for the two-point function. The orange square denotes $\partial_{\tau} R_{\tau}(x, y)$. Lines with blue circles represent the fully dressed propagator $G_{\tau}(x, y)=\mathrm{i}\left[\Gamma_{\tau}^{(2)}+R_{\tau}\right]^{-1}(x, y)$. The black dot with three legs is the classical vertex $S^{(3)}$. Since we further put $\Gamma_{\tau}^{(n)}=0$ for all $n>3$, all other diagrams vanish. perm. stands for the permutation of the external legs.

If we further insert (20) for the part including the regulator derivative, we obtain

$$
\partial_{\tau} \Gamma_{\tau}^{(2)}(x, y)=\frac{\mathrm{i} \lambda^{2}}{2} \partial_{\tau} G_{\tau}^{2}(x, y) .
$$

Integrating over $\tau$ is now straightforward. Due to our simple truncation, the flow (24) is in fact a total $\tau$ derivative. Let us stress again that the flow can also be integrated analytically in general truncations. This is discussed in detail in Appendix A and is a consequence of the causal structure of the flow. Performing the integration yields

$$
\Gamma^{(2)}(x, y)=\Gamma_{t_{0}}^{(2)}(x, y)+\frac{\mathrm{i} \lambda^{2}}{2} G^{2}(x, y),
$$

where $\Gamma_{t_{0}}^{(2)}$ is the free kinetic operator of (22). Thus, (25) can be solved as an integro-differential equation by applying it to the propagator $G$. This approach is very common in the literature. In our case, it yields the following equation:

$$
\begin{aligned}
& {\left[\partial_{x}^{2}+m^{2}\right] G(x, y)} \\
& \quad=-\mathrm{i} \delta_{\mathcal{C}}(x-y)+\frac{\mathrm{i} \lambda^{2}}{2} \int_{\mathcal{C}(z)} G^{2}(x, z) G(z, y) .
\end{aligned}
$$

Let us remark that Eq. (26) can also be obtained as the lowest order in 2PI-perturbation theory. The t-fRG framework can reproduce 2PI approximations by a suitable choice of truncation, cf. the discussion in [7,8] regarding the s-channel resummation. In general, however, the t-fRG framework allows for resummations that do not correspond to $2 \mathrm{PI}$ resummations, e.g., by including $\mathrm{t}$ - and $\mathrm{u}$-channel contributions of the four-point function. These options will be explored in a future work.

Another possibility is to invert the free kinetic operator $\Gamma_{t_{0}}^{(2)}$ using

$$
\int_{\mathcal{C}(z)} \Gamma_{t_{0}}^{(2)}(x, z) \bar{G}(z, y)=\mathrm{i} \delta_{\mathcal{C}}(x-y),
$$

where we denoted the inverse by $\bar{G}$. Since $\bar{G}$ is just the solution of the free equation of motion, it is known analytically. Multiplying (25) with $\bar{G}$ from the left and $G$ from the right, we obtain the integral equation

$G(x, y)=\bar{G}(x, y)-\frac{\lambda^{2}}{2} \int_{\mathcal{C}\left(z_{1}, z_{2}\right)} \bar{G}\left(x, z_{1}\right) G^{2}\left(z_{1}, z_{2}\right) G\left(z_{2}, y\right)$.

We also remark that while (28) seemingly is an implicit equation, it is in fact explicit for $x^{0} \geq y^{0}$. Using the symmetry of the propagator, this allows us to solve (28) explicitly without iterating it.

Let us briefly address the numerical solution of these equations. At a first glance, the integro-differential version of the equation can be solved faster since there is one time integral less compared to the integral version. However, demanding that the results have the same accuracy, this changes. Due to the derivative, a higher resolution is needed to achieve the same accuracy as with the integral equation. More details can be found in Appendix D.

\section{B. Results}

For the solution of the integrated flow, it is useful to formulate all equations in terms of the statistical two-point function $F$ and the spectral function $\rho$. These are defined as the expectation value of the anticommutator and commutator of the field, respectively, and (for $\phi=0$ ) read

$$
\begin{aligned}
F(x, y) & =\frac{1}{2}\langle\{\varphi(x), \varphi(y)\}\rangle, \\
\rho(x, y) & =\mathrm{i}\langle[\varphi(x), \varphi(y)]\rangle .
\end{aligned}
$$

From this definition, it is clear that $F$ is symmetric, while $\rho$ is antisymmetric. They are related to the propagator by

$$
G(x, y)=F(x, y)-\frac{\mathrm{i}}{2} \rho(x, y) \operatorname{sgn}_{\mathcal{C}}\left(x^{0}, y^{0}\right),
$$

where $\operatorname{sgn}_{\mathcal{C}}\left(x_{0}-y^{0}\right)$ is 1 for $x^{0}>y^{0}$ on the CTP and -1 for $x^{0}<y^{0}$.

The propagator already allows us to discuss relevant observables such as the occupation number and the dispersion relation. In nonequilibrium situations, there is no unique definition, but we can define versions analogously to the ones in equilibrium. To that end, we make use of the decomposition of the equal time statistical propagator; see, e.g., [40],

$$
F(t, t, \mathbf{p})=\frac{f(t, \mathbf{p})+\frac{1}{2}}{\omega(t, \mathbf{p})} .
$$




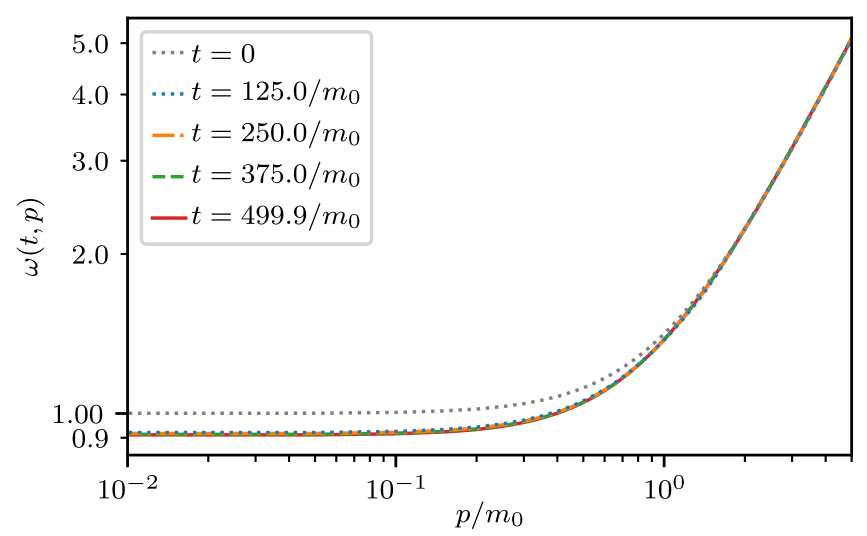

FIG. 7. Time evolution of the dispersion relation as defined in (33). It is depicted by showing the dispersion relation for various times. The gray dashed line corresponds to the dispersion at the initial time. At zero momentum, one can read off the mass of the interacting theory to be $m \approx 0.9 m_{0}$ relative to the bare mass.

The nonequilibrium generalizations of the occupation number $f(t, \mathbf{p})$ and dispersion relation $\omega(t, \mathbf{p})$ are chosen such that they coincide with their time-independent counterparts in equilibrium. The occupation number can be computed as

$$
f(t, \mathbf{p})=\left[\left.\partial_{t} \partial_{t^{\prime}} F\left(t, t^{\prime} ; \mathbf{p}\right)\right|_{t^{\prime}=t} F(t, t ; \mathbf{p})\right]^{1 / 2}-\frac{1}{2},
$$

and for the dispersion relation one finds

$$
\omega(t, \mathbf{p})=\left(\frac{\left.\partial_{t} \partial_{t^{\prime}} F\left(t, t^{\prime} ; \mathbf{p}\right)\right|_{t^{\prime}=t}}{F(t, t ; \mathbf{p})}\right)^{1 / 2} .
$$

In Appendix B, we exemplify the above definitions with the solution of the free equation of motion. There, one can directly verify that the definitions (32) and (33) give the desired results.

For the results shown in this section, we choose initial conditions far from equilibrium: we prepare a system with highly over-occupied momentum modes at small momenta and none for high momenta. Explicitly, we consider a (sharp) box for the initial occupancies $f_{0}(\mathbf{p})$ of the form

$$
f_{0}(\mathbf{p})=\frac{\tilde{N}}{\tilde{\lambda}} \theta\left(m_{0}-|\mathbf{p}|\right)
$$

Here, $\tilde{N}=100$ and the dimensionless coupling of the three-point function is given by $\tilde{\lambda}=\lambda / m_{0}^{2}=0.01$. More details on the initial conditions for the different solvers are given in Appendix B. The results of this section were obtained solving the integro-differential version of the equation.

A first interesting result is the time evolution of the dispersion relation shown in Fig. 7 for different times. At small momenta, the dispersion decreases with time. This region is dominated by the mass. For zero momentum, we

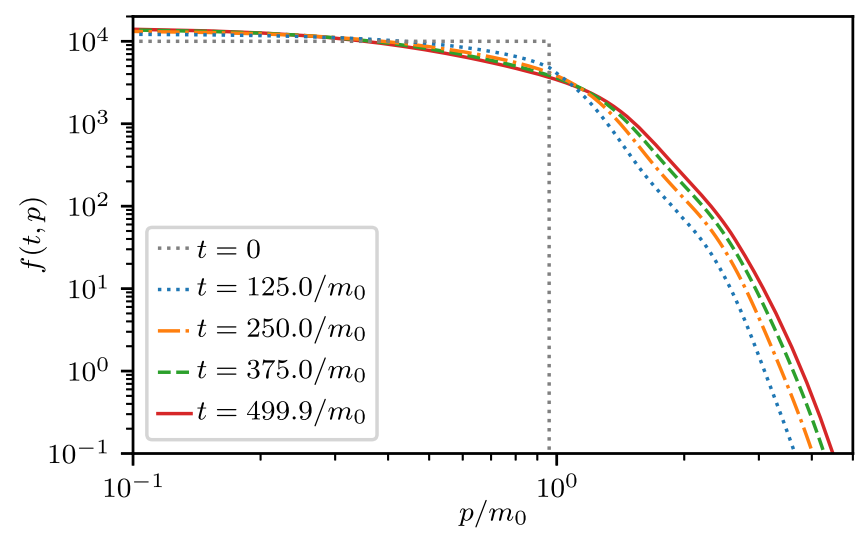

FIG. 8. Time evolution of the occupation number as defined in (32). It is represented by showing the occupation number for various times. The gray dashed line corresponds to the initial occupations. For later times, the initial box is smoothed out and we find indications for a self-similar scaling regime around $p / m_{0} \approx 2$ exhibiting a power law decay.

can therefore read off the mass $m$ of the interacting particles compared to the bare mass $m_{0}$ and find $m \approx 0.9 m_{0}$. For higher momenta, where the mass is negligible, the dispersion agrees for all times.

The time evolution of the occupation number is shown in Fig. 8 for the same times as used for the dispersion relation. Naturally, the initial sharp box is softened during the time evolution, and particles are redistributed over the range of momenta.

The momentum regime around $p / m_{0} \approx 2$ is particularly interesting. In this regime, we may identify self-similar scaling with a power law decay of the occupation number

$$
f(t, \mathbf{p}) \propto|\mathbf{p}|^{-\kappa} .
$$

For an estimate of the exponent $\kappa$, we compute the momentum-dependent exponent

$$
\kappa(t, p)=-p \partial_{p} \ln f(t, p) .
$$

This exponent is shown for different times in Fig. 9. In the momentum range $p / m_{0} \in[1.8,2.1]$, this exponent is approximately constant. At later times, this constant scaling regime is more pronounced, and we have evaluated the exponent at $t=499.9 / m_{0}$ for the momentum range above as

$$
\kappa \in[5.57,5.69], \quad \frac{p}{m_{0}} \in[1.8,2.1] .
$$

The analysis above suggests a power law behavior. Moreover, the exponent is similar for all times considered. This indicates a self-similar scaling, although the regime is rather small.

In regimes with self-similar scaling, the time evolution is characterized by a self-similar scaling of the occupancies; see, e.g., $[3,28]$. This scaling reads 


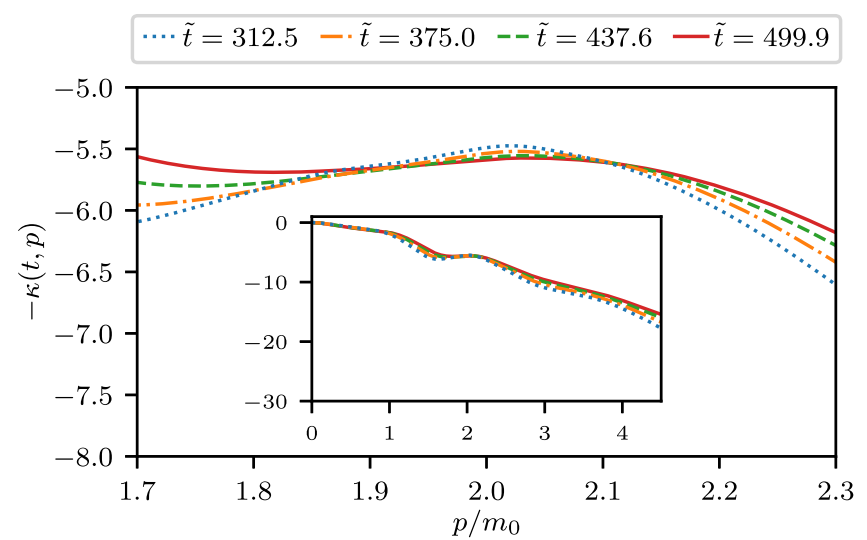

FIG. 9. Momentum-dependent exponents of the occupation number for various times $\tilde{t}=t m_{0}$ as defined in (36). In the momentum range $p / m_{0} \in[1.8,2.1]$, the exponents are approximately constant. At later times, this constant regime is more pronounced, and we have evaluated the exponent at $t=$ $499.9 / m_{0}$ as $\kappa \in[5.57,5.69]$. The inset shows the momentumdependent exponents for the full available momentum range.

$$
f\left(t_{\text {ref }},|\mathbf{p}|\right)=\left(\frac{t}{t_{\text {ref }}}\right)^{-\alpha} f\left[t,\left(\frac{t}{t_{\text {ref }}}\right)^{-\beta}|\mathbf{p}|\right] .
$$

In the regime $p / m_{0} \in[1.8,2.1]$, we find for the times $t m_{0}=312.5,375.0,437.6$ the exponents $\alpha=0.82,1.03$, 1.39 and $\beta=-0.02,0.02,0.09$, employing a least squares fit with respect to the occupancies at the reference time $t_{\text {ref }}=499.9 / m_{0}$. Figure 10 shows the original occupation numbers for the above times and Fig. 11 the ones rescaled each by their corresponding exponents. The rescaled occupation numbers match in the momentum range found from the power law exponent in accordance with a selfsimilar time evolution.

Next, we discuss the nontrivial and important consistency check of energy conservation for the present computation. The total energy is obtained by computing the expectation value of the time-time component of the energy-momentum

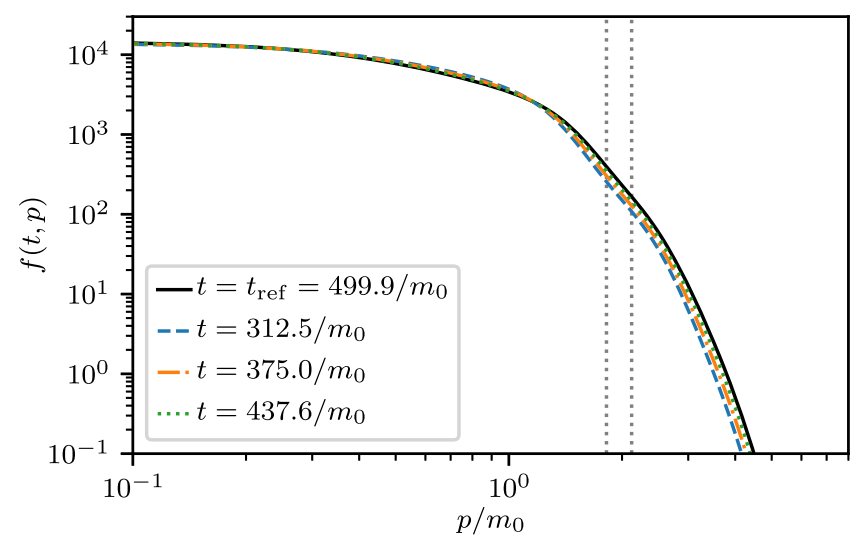

FIG. 10. Occupation numbers for times for which a self-similar time evolution is indicated in the momentum range marked by vertical dashed lines.

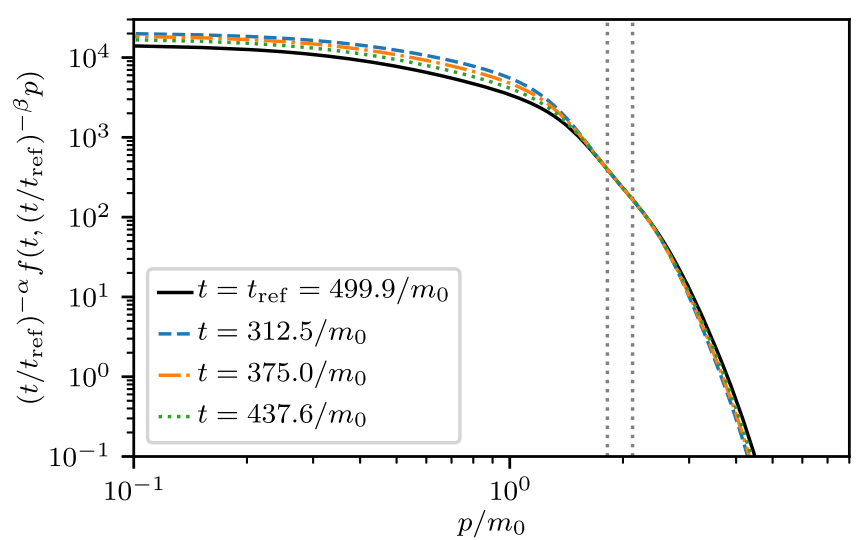

FIG. 11. Occupation numbers rescaled according to (38) for an assumed self-similar time evolution in the momentum range $p / m_{0} \in[1.8,2.1]$. This regime is marked by vertical dashed lines. The $\alpha, \beta$ exponents computed for times $t m_{0}=312.5$, $375.0,437.6$ are given by $\alpha=0.82,1.03,1.39$ and $\beta=-0.02$, $0.02,0.09$.

tensor $T_{\mu \nu}$. The details of this computation are discussed in Appendix $\mathrm{C}$, where a representation of the energy solely in terms of the propagator is derived; see (C8). Using $\rho(t, t, \mathbf{p})=0$ in $(\mathrm{C} 8)$, we find for the total energy

$$
\begin{aligned}
E(t)= & \left\langle T_{00}(t)\right\rangle=\lim _{t \rightarrow t^{\prime}} \frac{5}{6} \partial_{t} \partial_{t^{\prime}} \int_{\mathbf{p}} F\left(t, t^{\prime} ; \mathbf{p}\right) \\
& +\frac{1}{6} \int_{\mathbf{p}}\left(\mathbf{p}^{2}+m_{0}^{2}\right) F(t, t ; \mathbf{p}) .
\end{aligned}
$$

The explicit occurrence of $\Gamma^{(3)}$ has dropped out in (39) thanks to the structure of the gap equation (DysonSchwinger equation) for the two-point function (C7) in $\phi^{3}$ theory.

As was already mentioned, the truncation employed in the present work corresponds to the lowest order in 2PIperturbation theory. The latter is known to maintain energy conservation. However, this may be violated in numerical implementations. As a consistency check, we show the relative error of the total energy in Fig. 12. The error stabilizes at around $10^{-4}$ and the total energy is conserved.

While an analytical proof of energy conservation for general t-fRG truncations is lacking, the causal and local nature of the temporal flow provide a handle to address the question of analytically identifying conserving truncations. This is an ongoing work [37].

For the discussion of particle number conservation, we use

$$
\Delta f(t)=\frac{\int_{p} f(t, p)-f(0, p)}{\int_{p}|f(t, p)-f(0, p)|},
$$

which measures the sum of positive and negative flows of particle numbers normalized to the difference of positive 


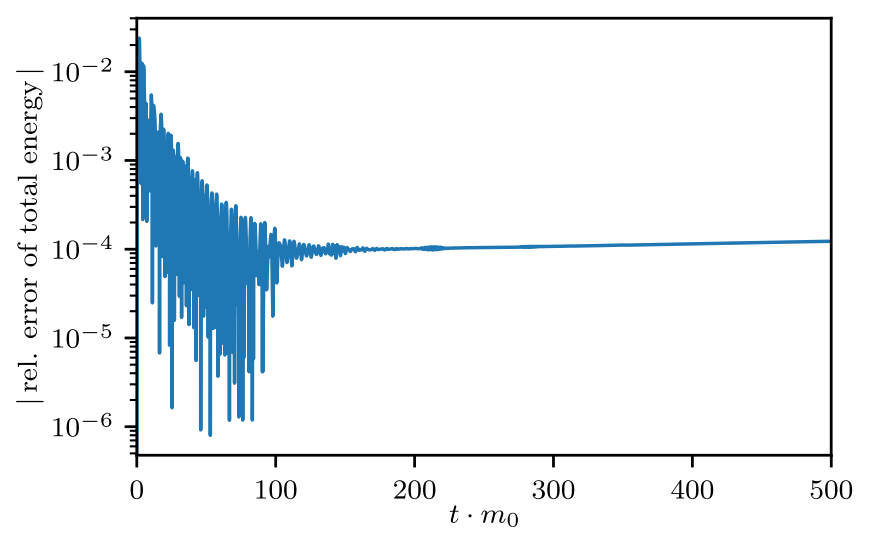

FIG. 12. Relative error of the total energy with respect to the initial total energy $|[E(t)-E(0)] / E(0)|$. After an initial tune in, the relative error stabilizes at around $10^{-4}$.

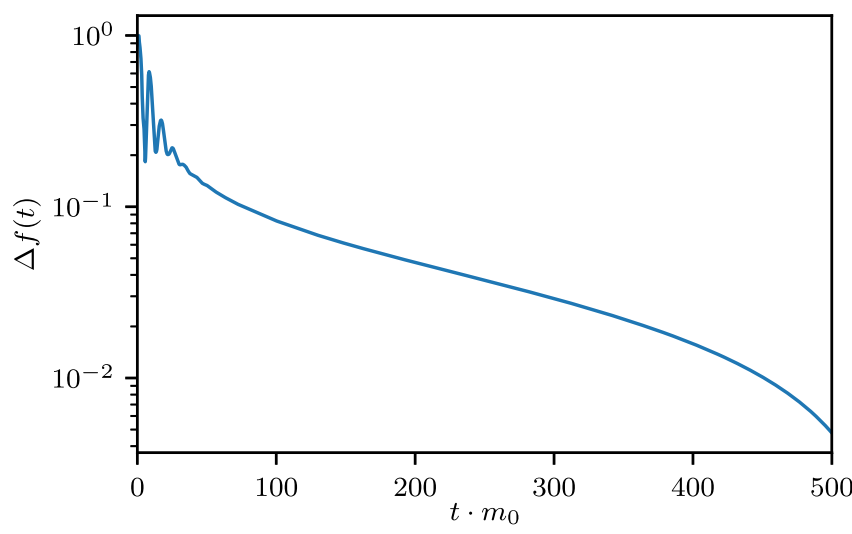

FIG. 13. Sum of positive and negative flows of the particle number normalized to the difference of positive and negative flows (total flow) of the particle number as defined in (40). After initial oscillations, the total particle number is conserved.

and negative flows of particle numbers (total flow). Figure 13 shows this quantity and we find, similarly to the total energy, that after the initial oscillations, the total particle number is conserved.

\section{CONCLUSION}

In the present work, we have further developed the temporal functional RG (t-fRG) suggested in [7,8]. By integrating the flow, we have derived novel one-loop exact functional relations for correlation functions. This result originates in the causal structure of the flow and its locality in time.

Moreover, we have applied the approach in its integrated form to the $\phi^{3}$ theory in $1+1$ dimensions. This theory serves as a test case for the approach and simulates the cubic interactions in a non-Abelian gauge theory. Here, we have studied the simplest approximation of the dynamics of the propagator, using classical vertices in the integrated flow. This approximation leads to a dynamical resummation of the propagator. We find indications of a scaling regime out of equilibrium with a momentum scaling $|\mathbf{p}|^{-\kappa}$ with $\kappa \in[5.57,5.69]$ at $t=499.9 / m_{0}$; see (37). An investigation of the self-similarity of the 3 times available led to the coefficients $\alpha \in[0.82,1.39]$ and $\beta \in[-0.02,0.09]$; see (38). Further investigations of this regime as well as the extension to situations with wave turbulence are deferred to future work.

There are several interesting extensions of the present work. The present framework allows for rather general causal approximation schemes, most notably the extension to nontrivial vertices and nonvanishing backgrounds. This is work in progress and we hope to report on the results in the near future.

\section{ACKNOWLEDGMENTS}

We thank Jürgen Berges, Thomas Gasenzer, Eduardo Grossi, Linda Shen, and Nicolas Wink for discussions. This work was supported by EMMI, the BMBF Grant No. 05P18VHFCA, and was part of and supported by the DFG Collaborative Research Centre SFB 1225 (ISOQUANT), as well as by the DFG under Germany's Excellence Strategy EXC-2181/1-390900948 (the Heidelberg Excellence Cluster STRUCTURES).

\section{APPENDIX A: INTEGRATED FLOW FOR THE PROPAGATOR}

Here, we discuss the analytic integration of the general flow equation of the two-point function with the help of causal relations. Remarkably, we obtain a one-loop exact functional relation valid for general theories. The derivation makes no use of the interaction structure of the theory. In particular, it applies to $\phi^{3}$ and $\phi^{4}$ theories used as explicitand general-examples in this section.

We start with the full flow of the two-point function (12). With (20), we replace $G_{\tau} \partial_{\tau} R_{\tau} G_{\tau}$ by $-\mathrm{i} \partial_{\tau} G_{\tau}$ and are led to

$$
\begin{aligned}
\partial_{\tau} \Gamma_{\tau}^{(2)}(x, y)= & \frac{1}{2} \int_{\mathcal{C}\left(z_{1}, z_{2}\right)} \Gamma_{\tau}^{(4)}\left(x, y, z_{1}, z_{2}\right) \partial_{\tau} G_{\tau}\left(z_{2}, z_{1}\right) \\
& +\frac{\mathrm{i}}{2} \int_{\mathcal{C}\left(z_{1}, \ldots, z_{4}\right)}\left[\Gamma_{\tau}^{(3)}\left(x, z_{1}, z_{2}\right) G_{\tau}\left(z_{2}, z_{4}\right)\right. \\
& \left.\times \Gamma_{\tau}^{(3)}\left(z_{3}, z_{4}, y\right) \partial_{\tau} G_{\tau}\left(z_{1}, z_{3}\right)+\text { perm. }\right] .
\end{aligned}
$$

From now on, we use a condensed notation with space-time arguments as indices. This makes the structural aspects of the following arguments more apparent. The first term on the right-hand side of (A1) can be rewritten as a total $\tau$ derivative and a $\partial_{\tau} \Gamma_{\tau}^{(4)}$ term, 


$$
\begin{aligned}
\frac{1}{2} \int_{\mathcal{C}\left(z_{1}, z_{2}\right)} \Gamma_{\tau, x y z_{1} z_{2}}^{(4)} \partial_{\tau} G_{\tau, z_{2} z_{1}}= & \partial_{\tau}\left[\frac{1}{2} \int_{\mathcal{C}\left(z_{1}, z_{2}\right)} \Gamma_{\tau, x y z_{1} z_{2}}^{(4)} G_{\tau, z_{2} z_{1}}\right] \\
& -\frac{1}{2} \int_{\mathcal{C}\left(z_{1}, z_{2}\right)} \partial_{\tau} \Gamma_{\tau, x y z_{1} z_{2}}^{(4)} G_{\tau, z_{2} z_{1}} .
\end{aligned}
$$

Equation (A2) relates to the fact that the flow equation for the effective action can be rewritten as a total $\tau$ derivative and an RG improvement term,

$$
\partial_{\tau} \Gamma_{\tau}=\frac{\mathrm{i}}{2} \operatorname{Tr} \partial_{\tau} \log \left[\Gamma_{\tau}^{(2)}+R_{\tau}\right]-\frac{1}{2} \operatorname{Tr} \partial_{\tau} \Gamma_{\tau}^{(2)} G_{\tau},
$$

where we have suppressed the field dependence. While such a rewriting is not required for applications, it carries much of the structure of the flow equation: typically, the first term is dominant, while the second term generates subleading RG improvements.

The first term in (A2) or (A3) can be integrated directly. For the second term, we use the causal properties of our approach and, in particular, (15) and (18). For $n=2$, we rewrite the causal property as

$$
\begin{aligned}
\partial_{\tau} \Gamma_{\tau, x y}^{(2)}= & \Delta \Gamma_{x y}^{(2)} *\left[\delta\left(\tau-x^{0}\right) \theta\left(\tau-y^{0}\right)\right. \\
& \left.+\theta\left(\tau-x^{0}\right) \delta\left(\tau-y^{0}\right)\right]
\end{aligned}
$$

with $\Delta \Gamma^{(2)}=\Gamma^{(2)}-\Gamma_{t_{0}}^{(2)}$; see (19). Here, $\Gamma_{t_{0}}$ is the input effective action. In the present example, $\Gamma_{t_{0}}^{(n)}$ are the $n$th derivatives of the classical action. The notation $\mathcal{O}_{1}(x, y) *$ $\mathcal{O}_{2}(x, y)$ takes account of the intricacy that $\partial_{\tau} \Gamma_{\tau, x y}^{(2)}$ may contain terms such as

$$
\lim _{r \rightarrow 0} \theta_{r}\left(\tau-x^{0}\right) \delta_{r}\left(\tau-x^{0}\right)=\frac{1}{2} \delta\left(\tau-x^{0}\right),
$$

where the subscript $r$ indicates a general regularization of the $\theta$ and $\delta$ functions. Terms as in the example (A5) arise from local contributions in $\Delta \Gamma^{(2)}$ proportional to $\delta_{\mathcal{C}}\left(x^{0}-z_{i}^{0}\right)$ with $i=1,2$. We emphasize that the product in (A5) is uniquely defined as both, the $\theta$ and the $\delta$ functions, share the same regularization as distributions, which originates in a regularization of the sharp temporal regulator $R_{\tau} \rightarrow R_{\tau, r}$.

This structure extends to general $n$-point functions and we write

$$
\Delta \Gamma_{x_{1} \cdots x_{n}}^{(n)}=\Delta \Gamma_{\mathrm{nl}, x_{1} \cdots x_{n}}^{(n)}+\Delta \Gamma_{\text {local }, x_{1} \cdots x_{n}}^{(n)},
$$

where we collect all terms that include $\delta_{\mathcal{C}}\left(x_{i}^{0}-x_{j}^{0}\right)$ with $i \neq j$ in the local part. We remark that for the nonlocal part, the $*$ product reduces to the standard product as no product of singular distributions is present.
Note that (A4) is proportional to $\delta\left(\tau-x^{0}\right)$ and $\delta\left(\tau-y^{0}\right)$. In the diagrams in (18), these are the external time arguments. Using (18) for $n=4$ and comparing with (A4), we conclude that there are two types of nonvanishing contributions of the second term of (A2) which are as follows:

(i) Contributions from the nonlocal part of the vertex: $\Delta \Gamma_{\mathrm{nl}}^{(4)}$ can only contribute if the $\tau$ derivative hits a $\theta$ function with an external time argument. If it hits an internal argument, the locality constraint of the flow is not satisfied and this contribution vanishes. This leads us to

$$
\begin{aligned}
& \Delta \Gamma_{\mathrm{nl}, x y z_{1} z_{2}}^{(4)}\left[\delta\left(\tau-x^{0}\right) \theta\left(\tau-y^{0}\right)+x^{0} \leftrightarrow y^{0}\right] \\
& \quad \times \theta\left(\tau-z_{1}^{0}\right) \theta\left(\tau-z_{2}^{0}\right),
\end{aligned}
$$

which carries the $\delta$ functions in (A4). The product in (A7) is the standard one.

(ii) Contributions from the local part of the vertex: $\Delta \Gamma_{\text {local }}^{(4)}$ contributions have to be computed with care. As for the first type in (A7), we receive contributions from $\partial_{\tau} \theta\left(\tau-x_{i}^{0}\right)$. Since additional factors $\theta\left(\tau-x_{i}^{0}\right)$ may be present, the $*$ product has to be evaluated $\left(i=1,2\right.$ and $\left.x_{1}^{0}=x^{0}, x_{2}^{0}=y^{0}\right)$.

Moreover, also contributions from the $\tau$ derivative of $\theta$ functions with an internal time argument $z_{j}^{0}$ have to be considered, as $\Delta \Gamma_{\text {local }}^{(4)}$ carries contributions with $\delta$ functions $\delta_{\mathcal{C}}\left(x_{i}^{0}-z_{j}^{0}\right)$ with $j=1,2$. These terms also carry the required causal structure. Again, the $*$ product has to be considered due to the potential occurrence of additional $\theta$ functions.

\section{Gap equation in the $\phi^{3}$ theory}

We now apply the above arguments to the two-point function of the $\phi^{3}$ theory used in the present work. Integrating the flow should result in the gap equation of the theory. While this is simply a consistency check of the approach, the locality and causality of the present framework will simplify the computation. We emphasize that these simplifications are also present in numerical applications.

To begin with, the $\phi^{3}$ theory has the important property

$$
\Delta \Gamma_{\text {local }}^{(n)} \equiv 0, \quad \forall n>2 .
$$

Equation (A8) greatly simplifies the following derivations and allows us to concentrate on some important aspects. For the sketchy proof of (A8) in the $\phi^{3}$ theory, we first consider the simplest vertex corrections for the three-point function, the triangle diagram with classical vertices. Thus, the vertex correction is given by

$$
\Delta \Gamma_{x_{1} x_{2} x_{3}}^{(3)} \propto \lambda^{3} G\left(x_{1}, x_{2}\right) G\left(x_{1}, x_{3}\right) G\left(x_{2}, x_{3}\right)+\cdots,
$$


where the dots stand for other diagrams as well as vertex corrections in the triangle. The space-time dependence of (A9) is simply given by a product of propagators. Evidently, this product does not contain temporal or spatial $\delta$ functions, as long as it is well defined.

We add that in dimensions $d \geq 1+5$, the product of propagators in (A9) is not well defined anymore at $x_{1}=x_{2}=x_{3}$. The respective terms are proportional to $\delta_{\mathcal{C}}\left(x_{1}-x_{2}\right) \delta_{\mathcal{C}}\left(x_{2}-x_{3}\right)$ and hence add to the classical coupling. This is nothing but the standard renormalization. A more detailed account of this will be presented elsewhere. In the present work, we consider $d=1+1$ and these intricacies are absent.

In summary, the vertex correction (A9) has no local pieces. Furthermore, any other vertex correction to the three-point function can be iteratively constructed from this diagram and the respective ones for the higher correlation functions. None of these diagrams can generate $\delta$ functions, as all legs are connected by propagators. In conclusion, the $n$-point functions in a $\phi^{3}$ theory have no local parts, except the initial (classical) vertex $\Gamma_{t_{0}}^{(3)}$.

This greatly simplifies the current investigation, as the local contributions are absent and no $*$ product has to be considered. Moreover, utilizing the manifest causality of the present approach will be pivotal for a simple derivation of the final result. Without loss of generality, we consider $\Gamma_{\tau}^{(2)}(x, y)$ for $x^{0}>y^{0}>t_{0}$. The full $\Gamma^{(2)}$ is obtained from

$\Gamma^{(2)}(x, y)=\Gamma_{t_{0}}^{(2)}(x, y)+\lim _{\epsilon \rightarrow 0_{+}} \int_{t_{0}}^{x^{0}+\epsilon} \mathrm{d} \tau \partial_{\tau} \Gamma_{\tau}^{(2)}(x, y)$.

The upper boundary in (A10) follows from the causality of the flow leading to $\partial_{\tau} \Gamma_{\tau}^{(2)}(x, y) \equiv 0$ for $\tau>x^{0}>y^{0}$. Due to causality, we could also move the lower boundary from $t_{0} \rightarrow x^{0}-\epsilon$. The infinitesimal shift with $\epsilon$ has been introduced as the flow has a contribution proportional to $\delta\left(\tau-x^{0}\right)$. Indeed, due to causality and locality, the flow is only nonvanishing for $\tau=x^{0}$. For $y^{0}>x^{0}$, the flow is only nonvanishing for $\tau=y^{0}$. Inserting the right-hand side of (A2) in (A10) leads us to a vanishing tadpole contribution,

$$
\begin{aligned}
& \frac{1}{2} \int_{\mathcal{C}\left(z_{1}, z_{2}\right) \leq x^{0}}\left[\Gamma_{x y z_{1} z_{2}}^{(4)} G_{z_{1} z_{2}}-\Gamma_{t_{0}, x y z_{1} z_{2}}^{(4)} G_{t_{0}, z_{1} z_{2}}-\Delta \Gamma_{x y z_{1} z_{2}}^{(4)} G_{z_{1} z_{2}}\right] \\
& \quad=0 .
\end{aligned}
$$

Equation (A11) follows from $\Gamma_{t_{0}}^{(4)}=0$ in the $\phi^{3}$ theory: accordingly, $\Delta \Gamma^{(4)}=\Gamma^{(4)}$ and the first and third terms cancel. The second term vanishes trivially: both $\Gamma_{t_{0}}^{(4)}$ and $G_{t_{0}}$ vanish separately.

We emphasize that the property (A11) is unique to the present t-fRG approach and is a consequence of both locality and causality. For other regulator choices and, in particular, the common momentum and frequency regulators, the tadpole contribution is nonvanishing. To obtain the gap equation from the integrated combined flow in such a setting, one has to insert the Dyson-Schwinger equation for the four-point function in the tadpole and proceed from there. Naturally, (A11) leads to great simplifications for numerical applications with more elaborate approximations.

Now, we proceed with the contributions from the threepoint functions in (A1). First, we use the symmetric occurrence of the propagators in the loop. This allows us to pull out the $\tau$ derivative in front of both propagators; schematically, this reads as $G_{\tau} \partial_{\tau} G_{\tau} \simeq(1 / 2) \partial_{\tau}\left(G^{2}\right)$ in the integral. Then we rewrite, similarly to the tadpole case, the three-point function contribution as a total $\tau$ derivative and $\partial_{\tau} \Gamma_{\tau}^{(3)}$ terms,

$$
\begin{aligned}
& \frac{\mathrm{i}}{2} \int_{\mathcal{C}\left(z_{1}, \ldots, z_{4}\right)}\left(\partial_{\tau}\left[\Gamma_{\tau, x z_{1} z_{2}}^{(3)} G_{\tau, z_{2} z_{4}} \Gamma_{\tau, z_{3} z_{4} y}^{(3)} G_{\tau, z_{1} z_{3}}\right]\right. \\
& \quad-\left(\partial_{\tau} \Gamma_{\tau, x z_{1} z_{2}}^{(3)}\right) G_{\tau, z_{2} z_{4}} \Gamma_{\tau, z_{3} z_{4} y}^{(3)} G_{\tau, z_{1} z_{3}} \\
& \left.\quad-\Gamma_{\tau, x z_{1} z_{2}}^{(3)} G_{\tau, z_{2} z_{4}}\left(\partial_{\tau} \Gamma_{\tau, z_{3} z_{4} y}^{(3)}\right) G_{\tau, z_{1} z_{3}}\right) .
\end{aligned}
$$

In $\partial_{\tau} \Gamma^{(3)}$, we only have to consider the parts proportional to $\delta\left(\tau-x^{0}\right)$ and $\delta\left(\tau-y^{0}\right)$, since the local terms are absent. Moreover, the term proportional to $\delta\left(\tau-y^{0}\right)$ vanishes as the cutoff time in this term, $\tau=y^{0}$, is smaller than the external time $x^{0}$. The first line is a total $\tau$ derivative and can be trivially integrated. In summary, the $\tau$ integration of (A12) leads us to

$$
\begin{aligned}
& \frac{\mathrm{i}}{2} \int_{\mathcal{C}\left(z_{1}, \ldots, z_{4}\right) \leq x^{0}}\left[\Gamma_{x z_{1} z_{2}}^{(3)}-\Delta \Gamma_{x z_{1} z_{2}}^{(3)}\right] G_{z_{2} z_{4}} \Gamma_{z_{3} z_{4} y}^{(3)} G_{z_{1} z_{3}} \\
& \quad=\frac{\mathrm{i}}{2} \int_{\mathcal{C}\left(z_{1}, \ldots, z_{4}\right) \leq x^{0}} \Gamma_{t_{0}, x z_{1} z_{2}}^{(3)} G_{z_{2} z_{4}} \Gamma_{z_{3} z_{4} y}^{(3)} G_{z_{1} z_{3}} .
\end{aligned}
$$

Equation (A13) is (the right-hand side of) the gap equation with an initial action $\Gamma_{t_{0}}$. In the present work, we use the classical action $S$ in (22) as the initial action. Thus, $\Gamma_{t_{0}}^{(3)}=$ $S^{(3)}$ and $\Gamma_{t_{0}}^{(2)}=S^{(2)}$. Then, (A13) reduces to the familiar gap equation

$$
\Gamma_{x y}^{(2)}-S_{x y}^{(2)}=-\frac{\mathrm{i} \lambda}{2} \int_{\mathcal{C}\left(z_{3}, z_{4}\right) \leq x^{0}} \Gamma_{z_{3} z_{4} y}^{(3)} G_{x z_{3}} G_{x z_{4}} .
$$

For the truncation in the present work with $\Gamma^{(3)}=S^{(3)}$, we directly obtain the integrated flow of our truncation (25) from (A14).

\section{Gap equation in the $\phi^{4}$ theory}

The lack of local terms in the $n$-point functions for the $\phi^{3}$ theory does not hold in general theories. While a full analysis is beyond the scope of the present work, the generic structure can be elucidated within the $\phi^{4}$ theory. 
From (A2), we get a generalized version of (A11) that takes the local contributions into account,

$$
\begin{aligned}
& \frac{1}{2} \int_{\mathcal{C}\left(z_{1}, z_{2}\right) \leq x^{0}}\left\{\Gamma_{t_{0}, x y z_{1} z_{2}}^{(4)} G_{z_{1} z_{2}}\right. \\
& \left.\quad+\Delta \Gamma_{\text {local }, x y z_{1} z_{2}}^{(4)} G_{z_{1} z_{2}}-\Delta \Gamma_{\text {local }, x y z_{1} z_{2}}^{(4)} * G_{z_{1} z_{2}}\right\} .
\end{aligned}
$$

The second term stems from the total derivative. We also keep the restriction $x^{0}>y^{0}>t_{0}$ already used in the previous section A 1 . Note also that the term at the initial cutoff $\tau=t_{0}$ still vanishes trivially despite $\Gamma_{t_{0}}^{(4)} \neq 0$ as $G_{t_{0}}(x, y)=0$. We emphasize that $\Delta \Gamma^{(4)} * G$ is a shorthand notation for $\int_{x^{0}-\epsilon}^{x^{0}+\epsilon} \partial_{\tau} \Delta \Gamma^{(4)} * G$.

Before we proceed with the computation, we discuss the diagrammatic relevance of the two terms in (A15): the first term can already be identified with a diagram in the gap equation, the tadpole with an initial four-point function. Naturally, it is absent in the $\phi^{3}$ theory. The second line is also nonvanishing, in contradistinction to the $\phi^{3}$ theory, as the vertex correction $\Delta \Gamma^{(4)}$ in the $\phi^{4}$ theory contains local parts. Indeed, one can show that all $n$-point functions contain local parts. This general structure will be considered elsewhere. Here, we elucidate this property with the simplest example relevant for the present discussion, the fish diagrams with classical vertices, which is the analog of (A9). It is self-consistently generated from the total derivative term in the fourth derivative of (A3) using the classical vertices. Then, the vertex correction of the fourpoint vertex is given by

$$
\begin{aligned}
\Delta \Gamma_{x y z_{1} z_{2}}^{(4)}= & \frac{\mathrm{i}}{2} \lambda_{4}^{2}\left[G^{2}(x, y) \delta_{\mathcal{C}}\left(x-z_{1}\right) \delta_{\mathcal{C}}\left(y-z_{2}\right)\right. \\
& +G^{2}(x, y) \delta_{\mathcal{C}}\left(x-z_{2}\right) \delta_{\mathcal{C}}\left(y-z_{1}\right) \\
& \left.+G^{2}\left(x, z_{1}\right) \delta_{\mathcal{C}}(x-y) \delta_{\mathcal{C}}\left(z_{1}-z_{2}\right)\right] \\
& +\cdots,
\end{aligned}
$$

where $\lambda_{4}$ is the initial (classical) coupling of the $\phi^{4}$ theory. In (A16), the dots stand for other diagrams as well as vertex corrections to the fish diagrams. The latter diagrams are evidently local. Inserting (A16) in the second line in (A15), we get flow contributions from the first two explicit terms in (A16).

The term directly proportional to $\delta_{\mathcal{C}}\left(x^{0}-y^{0}\right)$ leads to $2 \lim _{r \rightarrow 0} \theta_{r}\left(\tau-x^{0}\right) \delta_{r}\left(\tau-x^{0}\right)=\delta\left(\tau-x^{0}\right)$. Hence, this contribution cancels in the second line of (A15). In perturbation theory, this term potentially contributes to the "double tadpole," schematically given by $\delta_{\mathcal{C}}(x-y) G_{\mathrm{cl}, x z_{1}}^{2} G_{\mathrm{cl}, z_{1} z_{1}}$, where an integral over $z_{1}$ is implied. Here, $G_{\mathrm{cl}}$ is the classical propagator. However, in our approach, this term is contained completely in the first term in (A15). This is easily seen within a perturbative expansion of the propagator. It also follows directly from topological considerations of the diagrams in the gap equation: the first term in (A15) is a diagram in the gap equation and the other diagrams do not generate the double tadpole topology.

For the other terms, the $*$ product has to be evaluated. Thus, we have to consider the dependence on $\tau$. Each of the propagators $G(x, y)$ has to be multiplied with $\theta_{r}\left(\tau-x^{0}\right) \theta_{r}\left(\tau-y^{0}\right)$. We can ignore the second $\theta$ function as we only consider $x^{0}>y^{0}$. This leads to

$$
\partial_{\tau} \theta_{r}\left(\tau-x^{0}\right)^{2}=2 \delta_{r}\left(\tau-x^{0}\right) \theta_{r}\left(\tau-x^{0}\right)
$$

The contraction of $G_{z_{1} z_{2}}$ with the $\delta_{\mathcal{C}}\left(x_{i}^{0}-z_{j}^{0}\right)$ from (A16) provides further $2 \theta_{r}\left(\tau-x_{0}\right)$. Taking into account the factor $1 / 2$ from the vertex correction, cf. (A16), we arrive at

$$
2 \lim _{r \rightarrow 0} \theta_{r}\left(\tau-x^{0}\right)^{2} \delta_{r}\left(\tau-x^{0}\right)=\frac{2}{3} \delta\left(\tau-x^{0}\right) .
$$

The first term in the second line of (A15) contributes with a factor of 1 . The subtraction together with the global factor $1 / 2$ gives a combinatorial factor $1 / 6$,

$$
\frac{\mathrm{i}}{2}\left[\Delta \Gamma_{\text {local }}^{(4)} \cdot G-\Delta \Gamma_{\text {local }}^{(4)} * G\right]=\frac{\mathrm{i}}{6} \lambda_{4}^{2} G_{x y}^{3} .
$$

The right-hand side of (A19) is nothing but the sunset graph with full propagators, and the combinatorial factor is the correct one that can be deduced from perturbation theory. Notably, the vertex correction $\Delta \Gamma^{(4)}$ does not give contributions to the double tadpole diagram in perturbation theory. This is another unique property that stems from the present local and causal regulator.

In summary, we have seen how the local and causal structure can be used for computing the corrections. We also remark that the different powers of $\theta$ functions can be included systematically. For example, one easily obtains

$\lim _{r \rightarrow 0} f\left[\theta_{r}\left(\tau-x^{0}\right)\right] \delta_{r}\left(\tau-x^{0}\right)=\delta\left(\tau-x^{0}\right) \int_{0}^{1} d x f[x]$.

A detailed analysis is beyond the scope of the present work as no local terms are present in the $\phi^{3}$ theory. More details will be presented elsewhere.

Now, we proceed with the contributions of the threepoint functions in (A1). For $\phi=0$, they are absent in the $\phi^{4}$ theory but are present for $\phi \neq 0$ or in a theory with additional microscopic $\phi^{3}$ vertices. The general expression is obtained by simply adding the local terms to (A12) that were absent in the $\phi^{3}$ theory. We arrive at 


$$
\begin{aligned}
& \frac{\mathrm{i}}{2} \int_{\mathcal{C}\left(z_{1}, \ldots, z_{4}\right) \leq x^{0}}\left\{\Gamma_{t_{0}, x z_{1} z_{2}}^{(3)} G_{z_{2} z_{4}} \Gamma_{z_{3} z_{4} y}^{(3)} G_{z_{1} z_{3}}\right. \\
& \quad+\Delta \Gamma_{\text {local }, x z_{1} z_{2}}^{(3)}\left[G_{z_{2} z_{4}} \Gamma_{z_{3} z_{4} y}^{(3)} G_{z_{1} z_{3}}\right] \\
& \left.\quad-\Delta \Gamma_{\text {local }, x z_{1} z_{2}}^{(3)} *\left[G_{z_{2} z_{4}} \Gamma_{z_{3} z_{4} y}^{(3)} G_{z_{1} z_{3}}\right]\right\} .
\end{aligned}
$$

The first line in (A21) can already be identified with the respective term in the gap equation. Local terms for the correction of the three-point function are only generated from the $\phi^{4}$ interaction. We also remark that (A21) contains part of the squint diagram in the gap equation of the mixed theory with classical (initial) three- and four-point vertices $S^{(3)}$ and $S^{(4)}$. The rest of the squint diagram is generated by the respective vertex corrections to $\Delta \Gamma^{(4)}$ in the tadpole (A15). The squint diagram is the last missing diagram in the gap equation for the mixed theory.

Putting everything together, we are led to a remarkable novel result: a one-loop exact functional relation for the two-point function valid for general theories. We emphasize that other functional relations for correlation functions do not have a generic one-loop form; e.g., the DysonSchwinger equation for the propagator is only one-loop exact in the $\phi^{3}$ theory, while it is two-loop exact in the $\phi^{4}$ theory. The integrated flow of the two-point function for $x^{0} \geq y^{0}$ is given by

$$
\begin{aligned}
\Gamma_{x y}^{(2)}-\Gamma_{t_{0}, x y}^{(2)}= & \frac{1}{2} \int_{\mathcal{C}\left(z_{1}, z_{2}\right) \leq x^{0}}\left\{\Gamma_{t_{0}, x y z_{1} z_{2}}^{(4)} G_{z_{1} z_{2}}\right. \\
& \left.+\left(\Delta \Gamma_{\text {local }, x y z_{1} z_{2}}^{(4)}-\Delta \Gamma_{\text {local }, x y z_{1} z_{2}}^{(4)} *\right) G_{z_{1} z_{2}}\right\} \\
& +\frac{\mathrm{i}}{2} \int_{\mathcal{C}\left(z_{1}, \ldots, z_{4}\right) \leq x^{0}}\left\{\Gamma_{t_{0}, x z_{1} z_{2}}^{(3)} G_{z_{2} z_{4}} \Gamma_{z_{3} z_{4} y}^{(3)} G_{z_{1} z_{3}}\right. \\
& +\left(\Delta \Gamma_{\text {local }, x z_{1} z_{2}}^{(3)}-\Delta \Gamma_{\text {local }, x z_{1} z_{2}}^{(3)} *\right) \\
& \left.\times\left[G_{z_{2} z_{4}} \Gamma_{z_{3} z_{4} y}^{(3)} G_{z_{1} z_{3}}\right]\right\} .
\end{aligned}
$$

The terms on the right-hand side involving the vertices at $t_{0}$ are the one-loop diagrams in the gap equation; the other terms generate two-loop terms. As mentioned before, this one-loop exact relation is the general result for generic theories independent of the initial action. Using the same reasoning, $\partial_{\tau} \Gamma_{\tau}^{(n)}$ can be integrated analytically for any $n$. We close with the remark that this is only seemingly in contradiction with the proof that such one-loop exact functional relations do not exist in [41]. The present approach implicitly escapes one of the presuppositions there (no integral over parameters such as the cutoff time) via (A20).

\section{APPENDIX B: INITIAL CONDITIONS}

We employ a mixed position and momentum space, where only the spatial coordinates are replaced by momenta.
In case of using the integral equation (28), the initial conditions are encoded in the solution of the free equation of motion $\bar{G}$, or $\bar{F}$ and $\bar{\rho}$,

$$
\begin{aligned}
& \bar{F}\left(t, t^{\prime} ; \mathbf{p}\right)=\frac{1}{\omega_{\mathbf{p}}}\left(\frac{1}{2}+f_{0}(\mathbf{p})\right) \cos \left[\omega_{\mathbf{p}}\left(t-t^{\prime}\right)\right], \\
& \bar{\rho}\left(t, t^{\prime} ; \mathbf{p}\right)=\frac{1}{\omega_{\mathbf{p}}} \sin \left[\omega_{\mathbf{p}}\left(t-t^{\prime}\right)\right] .
\end{aligned}
$$

Here, $f_{0}(\mathbf{p})$ is the occupation number and $\omega_{\mathbf{p}}=\sqrt{\mathbf{p}^{2}+m_{0}^{2}}$ the dispersion relation at initial time.

For the differential equation approach (26), we have to provide values for $F$ and $\rho$ and their first derivatives at the initial time. In analogy to the previous case, we use for the statistical two-point function

$$
\begin{aligned}
\left.F\left(t, t^{\prime} ; \mathbf{p}\right)\right|_{t=t^{\prime}=t_{0}} & =\frac{1}{\omega_{\mathbf{p}}}\left(\frac{1}{2}+f_{0}(\mathbf{p})\right), \\
\left.\partial_{t} F\left(t, t^{\prime} ; \mathbf{p}\right)\right|_{t=t^{\prime}=t_{0}} & =0 \\
\left.\partial_{t} \partial_{t^{\prime}} F\left(t, t^{\prime} ; \mathbf{p}\right)\right|_{t=t^{\prime}=t_{0}} & =\omega_{\mathbf{p}}\left(\frac{1}{2}+f_{0}(\mathbf{p})\right)
\end{aligned}
$$

and the spectral function

$$
\begin{aligned}
\left.\rho\left(t, t^{\prime} ; \mathbf{p}\right)\right|_{t=t^{\prime}=t_{0}} & =0, \\
\left.\partial_{t} \rho\left(t, t^{\prime} ; \mathbf{p}\right)\right|_{t=t^{\prime}=t_{0}} & =1, \\
\left.\partial_{t} \partial_{t^{\prime}} \rho\left(t, t^{\prime} ; \mathbf{p}\right)\right|_{t=t^{\prime}=t_{0}} & =0 .
\end{aligned}
$$

For the results shown in Sec. III B, we used

$$
f_{0}(\mathbf{p})=\frac{\tilde{N}}{\tilde{\lambda}} \theta\left(m_{0}-|\mathbf{p}|\right),
$$

with $\tilde{N}=100$. The dimensionless coupling of the threepoint function is given by $\tilde{\lambda}=\lambda / m_{0}^{2}=0.01$.

\section{APPENDIX C: TOTAL ENERGY}

The total energy is given by $\left\langle T_{00}\right\rangle$ and the expectation value of the energy-momentum tensor is obtained from the effective action as

$$
\left\langle T_{\mu \nu}(x)\right\rangle=\left.\frac{2}{\sqrt{-g(x)}} \frac{\delta \Gamma[\phi, g]}{\delta g^{\mu \nu}(x)}\right|_{g^{\mu \nu}=\eta^{\mu \nu}} .
$$

In (C1), the metric $g^{\mu \nu}$ is identified with the Minkowski metric $\eta^{\mu \nu}$. The flow of $\left\langle T_{\mu \nu}\right\rangle$ can be derived from the metric variation of $\partial_{\tau} \Gamma_{\tau}$ and will be discussed elsewhere. Here, we follow closely derivations also found in the $2 \mathrm{PI}$ framework, e.g., [38], which allows us to discuss the diagrammatical consistency of our truncation. 
Concentrating on $\left\langle T_{00}\right\rangle$, we use that $T_{00}$ is derived from (C1) by substituting $\Gamma$ by the classical action (22). This leads us to

$T_{00}(x)=\partial_{0} \varphi \partial_{0} \varphi-g_{00}\left(\frac{1}{2} \partial_{\mu} \varphi \partial^{\mu} \varphi-\frac{1}{2} m_{0}^{2} \varphi^{2}-\frac{\lambda}{3 !} \varphi^{3}\right)$.

For its expectation value, we use that (see, e.g., [35])

$$
\left\langle\prod_{i=1}^{n} \varphi\left(x_{i}\right)\right\rangle=\prod_{i=1}^{n}\left[\int_{\mathcal{C}\left(z_{i}\right)} G\left(x_{i}, z_{i}\right) \frac{\delta}{\delta \phi\left(z_{i}\right)}+\phi\left(x_{i}\right)\right]_{\phi_{\mathrm{EoM}}},
$$

with the full mean-field-dependent propagators $G[\phi](x, y)$ and the mean field $\phi=\langle\varphi\rangle$ being evaluated on the equations of motion (EoM). In the present case, we have $\phi_{\text {EoM }}=0$. Thus, $\left\langle T_{00}\right\rangle$ reduces to

$$
\begin{aligned}
\left\langle T_{00}(x)\right\rangle= & \frac{1}{2} \lim _{y \rightarrow x}\left[\partial_{x^{0}} \partial_{y^{0}} G(x, y)+\left(-\partial_{\mathbf{x}}^{2}+m_{0}^{2}\right) G(x, y)\right] \\
& +\frac{\mathrm{i} \lambda}{3 !} \int_{\mathcal{C}\left(z_{1}, z_{2}, z_{3}\right)} \Gamma^{(3)}\left(z_{1}, z_{2}, z_{3}\right) \prod_{i=1}^{3} G\left(x, z_{i}\right),
\end{aligned}
$$

where we have used

$\frac{\delta}{\delta \phi\left(z_{1}\right)} G(x, y)=\mathrm{i} \int_{\mathcal{C}\left(z_{2}, z_{3}\right)} G\left(x, z_{2}\right) \Gamma^{(3)}\left(z_{1}, z_{2}, z_{3}\right) G\left(z_{3}, y\right)$.

The last term in (C4) is the vacuum sunset term. Its oneloop subgraph is related to the gap equation (DysonSchwinger equation)

$$
\begin{aligned}
& \Gamma^{(2)}(x, y) \\
& \quad=S^{(2)}(x, y)-\frac{\mathrm{i} \lambda}{2} \int_{\mathcal{C}\left(z_{1}, z_{2}\right)} \Gamma^{(3)}\left(y, z_{1}, z_{2}\right) G\left(z_{1}, x\right) G\left(z_{2}, x\right),
\end{aligned}
$$

which leads us to

$$
\begin{aligned}
& \frac{\mathrm{i} \lambda}{3 !} \int_{\mathcal{C}\left(z_{1}, z_{2}, z_{3}\right)} \Gamma^{(3)}\left(z_{1}, z_{2}, z_{3}\right) \prod_{i=1}^{3} G\left(x, z_{i}\right) \\
& \quad=\frac{1}{3} \int_{\mathcal{C}(z)}\left[S^{(2)}(x, z)-\Gamma^{(2)}(x, z)\right] G(z, x) .
\end{aligned}
$$

The second term is proportional to an irrelevant constant, $\left(\Gamma^{(2)} \cdot G\right)(x, y)=\mathrm{i} \delta_{\mathcal{C}}(x-y)$, while the first one simply changes the prefactors of the first and second term on the right-hand side of $(\mathrm{C} 4)$. Fourier transforming the spatial coordinates, we arrive at

$$
\left\langle T_{00}(t)\right\rangle=\frac{5}{6} \lim _{t \rightarrow t^{\prime}} \partial_{t} \partial_{t^{\prime}} \int_{\mathbf{p}} G\left(t, t^{\prime} ; \mathbf{p}\right)+\frac{1}{6} \int_{\mathbf{p}}\left(\mathbf{p}^{2}+m_{0}^{2}\right) G(t, t ; \mathbf{p}) .
$$

Note that the explicit occurrence of $\Gamma^{(3)}$ has dropped out in (C8). This trivially ensures the self-consistency of (C8) with the approximation of $\Gamma^{(3)}$ used in the computation of the propagator. We also remark that $(\mathrm{C} 8)$ readily extends to all components of the expectation value of the energymomentum tensor $\left\langle T_{\mu \nu}\right\rangle$.

\section{APPENDIX D: NUMERICAL DETAILS}

The initial conditions for the results shown in this section can be found in Appendix B. The parameters used are the same as in the rest of the paper [cf. (B4) and below].

To solve the integro-differential equation (26), we use a symmetric discretization of the second-order time derivative. This allows us to use the simple explicit Euler method to compute the solution. More details can be found, e.g., in [40]. All integrals are computed using a trapezoidal rule.

In Fig. 14, we show the solution for statistical propagator $F\left(0, t, p=4.04 m_{0}\right)$ for three different time-step sizes $\Delta \tilde{t}=\Delta t \cdot m_{0}$. For a decreasing step size, the curves get closer to each other. We do not observe an instability for the step sizes used.

In Fig. 15, we show $F\left(0, t, p=4.04 m_{0}\right)$ as obtained from the integral equation (28) for the same times as in Fig. 14 and the same step sizes. We observe that the curves are perfectly on top of each other for all shown step sizes. Thus, the explicit solver obtained from the discretization of the integral equation (28) converges faster than the differential one. Details on how to solve (28) as an explicit equation will be given elsewhere.

What remains to be shown is that both methods agree for sufficiently small step sizes. This can be seen from Fig. 16 where we compare both solvers for the smallest available

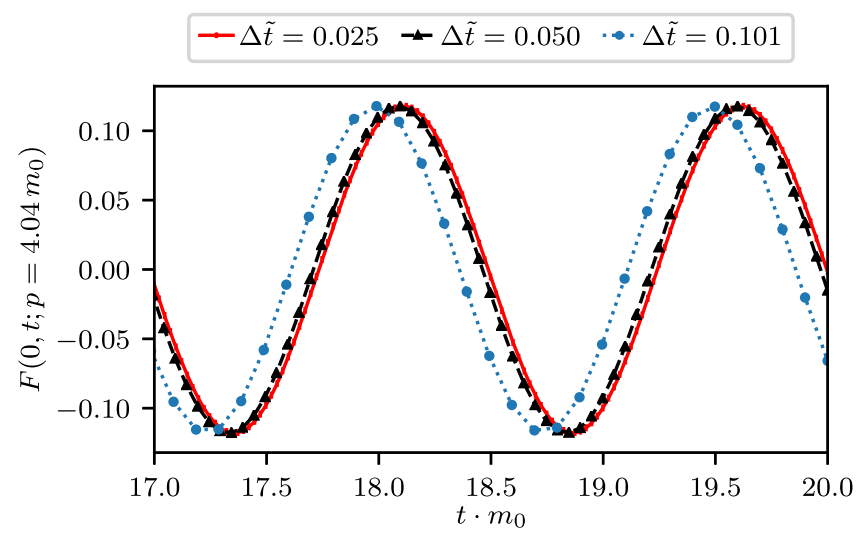

FIG. 14. Time evolution of the statistical propagator obtained using the differential solver for various time resolutions $\Delta \tilde{t}=\Delta t \cdot m_{0}$. The curves get closer to each other for decreasing time-step size. 


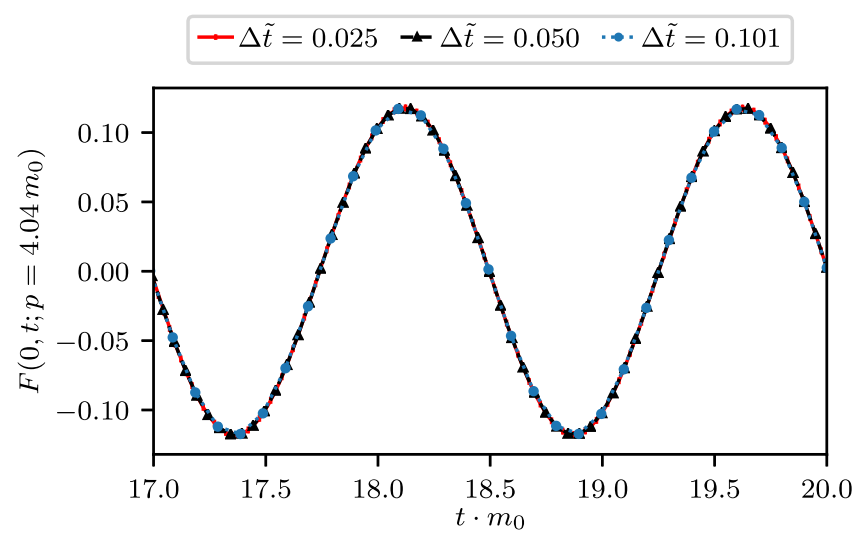

FIG. 15. Time evolution of the statistical propagator obtained using the explicit solver for various time resolutions $\Delta \tilde{t}=\Delta t \cdot m_{0}$. The curves are perfectly on top of each other. This demonstrates the faster convergence of the explicit solver as compared to the differential one.

step size. The same agreement is obtained comparing the largest one for the explicit solver with the smallest one of the differential solver.

Taking a closer look at Fig. 16, we see that the black dashed line is still slightly shifted compared to the red one. This again demonstrates the faster convergence of the explicit solver.

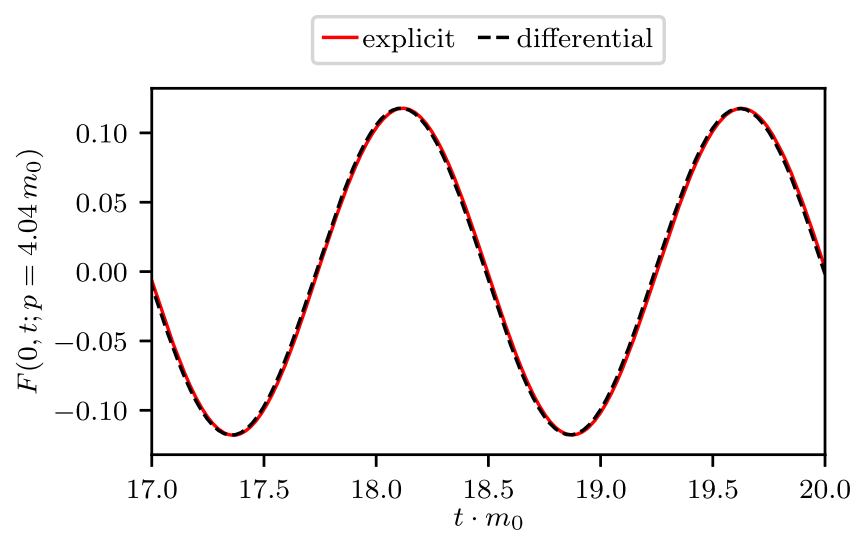

FIG. 16. Time evolution of the statistical propagator obtained using the differential and explicit solver for $\Delta \tilde{t}=$ $\Delta t \cdot m_{0}=0.025$. Both solvers agree for small enough timestep size.

The faster convergence comes at the price of an additional time integral that has to be computed. However, the differential solver requires a smaller time-step size to produce results of the same accuracy. For the results shown in this section for $\Delta \tilde{t}=0.025$ for the differential solver and $\Delta \tilde{t}=0.101$ for the explicit solver, the runtime of both solvers is comparable (same order of magnitude).
[1] B. Nowak, S. Erne, M. Karl, J. Schole, D. Sexty, and T. Gasenzer, arXiv:1302.1448.

[2] J. Berges, arXiv:1503.02907.

[3] C.-M. Schmied, A. N. Mikheev, and T. Gasenzer, Int. J. Mod. Phys. A 34, 1941006 (2019).

[4] C. Wetterich, Phys. Lett. B 301, 90 (1993).

[5] U. Ellwanger, Z. Phys. C 62, 503 (1994).

[6] T. R. Morris, Int. J. Mod. Phys. A 09, 2411 (1994).

[7] T. Gasenzer and J. M. Pawlowski, Phys. Lett. B 670, 135 (2008).

[8] T. Gasenzer, S. Kessler, and J. M. Pawlowski, Eur. Phys. J. C 70, 423 (2010).

[9] M. Pietroni, J. Cosmol. Astropart. Phys. 10 (2008) 036.

[10] S. G. Jakobs, V. Meden, and H. Schoeller, Phys. Rev. Lett. 99, 150603 (2007).

[11] J. Berges and G. Hoffmeister, Nucl. Phys. B813, 383 (2009).

[12] S. G. Jakobs, M. Pletyukhov, and H. Schoeller, Phys. Rev. B 81, 195109 (2010).

[13] L. Canet, H. Chate, B. Delamotte, and N. Wschebor, Phys. Rev. Lett. 104, 150601 (2010).

[14] D. M. Kennes, S. G. Jakobs, C. Karrasch, and V. Meden, Phys. Rev. B 85, 085113 (2012).

[15] L. Canet, H. Chate, B. Delamotte, and N. Wschebor, Phys. Rev. E 84, 061128 (2011).
[16] J. Berges and D. Mesterhazy, Nucl. Phys. B, Proc. Suppl. 228, 37 (2012).

[17] L. M. Sieberer, S. D. Huber, E. Altman, and S. Diehl, Phys. Rev. Lett. 110, 195301 (2013).

[18] D. Mesterházy, J. H. Stockemer, L. F. Palhares, and J. Berges, Phys. Rev. B 88, 174301 (2013).

[19] L. M. Sieberer, S. D. Huber, E. Altman, and S. Diehl, Phys. Rev. B 89, 134310 (2014).

[20] L. Canet, B. Delamotte, and N. Wschebor, Phys. Rev. E 93, 063101 (2016).

[21] S. Mathey, T. Gasenzer, and J. M. Pawlowski, Phys. Rev. A 92, 023635 (2015).

[22] D. Mesterházy, J. H. Stockemer, and Y. Tanizaki, Phys. Rev. D 92, 076001 (2015).

[23] A. Chiocchetta, A. Gambassi, S. Diehl, and J. Marino, Phys. Rev. B 94, 174301 (2016).

[24] C. Duclut and B. Delamotte, Phys. Rev. E 95, 012107 (2017).

[25] M. Tarpin, L. Canet, and N. Wschebor, Phys. Fluids 30, 055102 (2018).

[26] M. Tarpin, L. Canet, C. Pagani, and N. Wschebor, J. Phys. A 52, 085501 (2019).

[27] H. Schoeller, arXiv:1802.10014.

[28] A. Piñeiro Orioli, K. Boguslavski, and J. Berges, Phys. Rev. D 92, 025041 (2015). 
[29] J. Schwinger, J. Math. Phys. (N.Y.) 2, 407 (1961).

[30] K. T. Mahanthappa, Phys. Rev. 126, 329 (1962).

[31] P. M. Bakshi and K. T. Mahanthappa, J. Math. Phys. (N.Y.) 4, 1 (1963).

[32] P. M. Bakshi and K. T. Mahanthappa, J. Math. Phys. (N.Y.) 4, 12 (1963).

[33] L. V. Keldysh, Zh. Eksp. Teor. Fiz. 47, 1515 (1964).

[34] L. Keldysh, Sov. Phys. JETP 20, 1018 (1965).

[35] J. M. Pawlowski, Ann. Phys. (Amsterdam) 322, 2831 (2007).
[36] L. Corell, M. Heller, and J. M. Pawlowski, Renormalised Causal Temporal Flow (to be published).

[37] M. Heller and J. M. Pawlowski, Causal Temporal Flow of the Energy-Momentum Tensor (to be published).

[38] J. Berges and B. Wallisch, Phys. Rev. D 95, 036016 (2017).

[39] L. Shen and J. Berges, Phys. Rev. D 101, 056009 (2020).

[40] J. Berges, AIP Conf. Proc. 739, 3 (2005).

[41] D. F. Litim and J. M. Pawlowski, Phys. Rev. D 66, 025030 (2002). 